\title{
Genetic and Epigenetic Aspects of Crouzon's Syndrome
}

\author{
Nasytha Vikarina Siregar ${ }^{1}$, Elza Ibrahim Auerkari ${ }^{2 *}$ \\ ${ }^{1}$ Orthodontic Residency Program, Faculty of Dentistry, University of Indonesia, Jakarta 10430, \\ Indonesia \\ ${ }^{2}$ Department of Oral Biology, Faculty of Dentistry, University of Indonesia, Jakarta 10430, \\ Indonesia \\ *Email: elza.ibrahim@ui.ac.id
}

\begin{abstract}
Crouzon's syndrome is a genetic disorder characterized by a distinctive malformation of the skull and facial region resulting from the premature fusion of the cranial sutures, abnormal growth of bones, and facial deformities caused by the underdevelopment of the midfacial region and hypertelorism. This syndrome is rare, with a prevalence of 16 cases among every million births worldwide. Mutations in the gene encoding fibroblast growth factor receptor 2 (FGFR2) have been identified as the main causative factors of autosomal dominant Crouzon's syndrome. However, Crouzon's syndrome may emerge as the result of a de novo mutation, in addition to its transmission as an autosomal dominant genetic condition. Crouzon's syndrome may also result from epigenetic mechanisms, such as a decrease in microRNA-338 expression that promotes increases FGFR2 expression and, consequently, enhanced osteoblast differentiation. This work aims to briefly review Crouzon's syndrome in the context of its genetic and epigenetic aspects, with the aim of allowing clinicians to more readily predict a patient's orthopedic condition before initiating orthodontic treatment.
\end{abstract}

Keywords: crouzon's syndrome, craniosynostosis, mutation, FGFR2 gene

\section{Introduction}

Craniosynostosis is a birth defect involving the premature fusion of one or more of the joints between the bones in the skull of an infant. This fusion occurs before the infant's brain has fully developed, leading to an abnormal skull shape happens. In Indonesia, the prevalence rate of craniosynostosis is 1 case per 2000 births, and most cases involve a single-suture synostosis affecting the sagittal or coronal sutures [1]. A genetic study identified FGFR2, FGFR3, TWIST1, and EFNB1 as the most frequently mutated genes associated with craniosynostosis [2]. Crouzon's syndrome, Pfeiffer syndrome, Apert syndrome, and Saethre-Chotzen syndrome have been identified as the most common types of craniosynostosis [3].

Of these, Crouzon's syndrome, a rare autosomal dominant inherited disorder with a prevalence of 16 cases per million births worldwide, is the most common type, accounting for $4.8 \%$ of all cases of craniosynostosis $[4,5]$. Notably, however, $50 \%$ of Crouzon's syndrome events are attributed to de novo, rather than inherited, mutations. The occurrence of Crouzon's syndrome has no sex or racial predilection and is unrelated to intrauterine drug exposure. 
Louis Edouard Octave Crouzon, a French neurologist, initially reported this syndrome for the first time in 1912 in a mother and son who presented with a triad of calvarial deformities, facial anomalies, and exophthalmos [3,7,8]. Craniosynostosis and facial hypoplasia are characteristic of Crouzon's syndrome, [9] and although the craniosynostosis type varies, most cases involve both coronal sutures. This syndrome is caused by the prenatal fusion of the superior and posterior sutures of the maxilla along the wall of the orbit. Underdevelopment of the midface and bulging of the eyes are also characteristic of Crouzon's syndrome, [10] and maxillary hypoplasia and mandibular prognathism with malocclusion often occur as a consequence of cranial base and midface suture involvement.11 Although nearly all patients with Crouzon's syndrome exhibit normal brain development, $13 \%$ exhibit mental retardation, $11.5 \%$ have a history of epilepsy, and $30 \%-50 \%$ develop severe recurrent headaches [12]. Furthermore, a syndrome-related disproportion between craniosynostosis and brain growth can cause a high and potentially fatal level of intracranial pressure.

Crouzon's syndrome can be distinguished from Apert syndrome by the tendency of the latter to manifest with an asymmetric cranial base, which is rare in the former because the skull is more mature at birth and the bones are fused to each other by multiple synostoses [13]. Other statistically significant differences include an increased lateral orbital wall angle, which is characteristic of Crouzon's syndrome. Moreover, sinusitis and nasal septum deviation almost always occur in patients with Crouzon's syndrome. Intraoral manifestations of Crouzon's syndrome include mandibular prognathism, maxillary hypoplasia with a narrow palate, and crowding, [14] as well as peg-shaped teeth, oglidontia, macrodontia, and widely spaced teeth. Patients with Crouzon's syndrome also frequently exhibit an imbalance of the extraocular muscles, as well as recurrent ear infections that lead to hearing loss.

\section{Genetics of Crouzon's syndrome}

Crouzon's syndrome is a rare genetic disorder caused by mutations in the fibroblast growth factor receptor $2(F G F R 2)$ gene $[6,8]$. This gene which has been identified on chromosome 10, encodes the FGFR2 protein, a tyrosine-protein kinase that acts as a cell-surface receptor for FGF $[15,18]$ FGFR2 belongs to the FGFR protein family, of which all four members play roles in important processes such as the regulation of cell growth and maturation, cell division, embryonic development, blood vessel formation, and wound healing.16 Specifically, FGFR2 plays essential regulatory roles in intracellular signaling pathways affecting cell proliferation, differentiation, migration, apoptosis, and embryonic development [17]. Among its multiple functions, FGFR2 protein signals immature cells to become bone cells during embryonic development.

Mutations in FGFR2 have been found to involve three extracellular immunoglobulin (Ig)-like C2-type domains (Ig-I, Ig-II, and Ig-III), a transmembrane domain, and a cytoplasmic tyrosine kinase domain. A genetic study found that nearly 60 FGFR2 mutations, most of which affected the Ig-III domain at locus 10q26.13, could cause Crouzon's syndrome [19]. Mutations in FGFR2 lead to the hyperactive signaling of FGFR2, which causes the bones of the skull to fuse prematurely. This 
premature fusion frequently extends posteriorly into the cranium to distort the cranial vault. Maxilla can prevent from translating downward and forward is the result of fusion in the orbital area. Once a suture has become fused, growth perpendicular to the suture is restricted, and the fused bones act as a single bony structure. Consequently, compensatory growth at the remaining open sutures causes abnormal bone growth and facial deformities while allowing continued brain growth.

\section{Epigenetics of Crouzon's syndrome}

The term "epigenetics" generally refers to changes in gene function that are mitotically heritable but do not involve a change in the DNA sequence. Epigenetics may be influenced by environmental factors such as histone modification, DNA methylation, and post-transcriptional silencing mediated by RNA interference, and these processes may enhance or repress gene expression. MicroRNAs (miRNAs) are small (approximately 21-24 nucleotides) noncoding RNAs that act as post-transcriptional regulators of gene expression [20]. These miRNAs have been identified in various biological processes, including cell differentiation, proliferation, and metabolism, and a loss of miRNA-mediated regulation can cause disease progression. MiRNAs can affect cell differentiation mediated by the FGF signaling pathway by directly regulating the expression of either FGF or FGFRs. For example, in osteoblasts, miR-338 can directly regulate the $3^{\prime}$ untranslated region of FGFR2 to suppress FGFR2 expression [20] Therefore, a decrease in miR-338 expression would enhance FGFR2 expression and, consequently, osteoblast differentiation. Moreover, miRNAs can affect FGF signaling during development by regulating downstream effectors of the related pathway [20].

\section{Conclusion}

Crouzon's syndrome is a genetic disorder characterized by the synostosis and premature fusion of coronal and sagittal sutures. The differential diagnosis of Crouzon's syndrome includes Apert, Pfeiffer, Carpenter, and Saethre-Chotzen syndromes. Mutations in the FGFR2 gene at chromosomal locations 10q25-26 have been implicated in the etiology of Crouzon's syndrome. These mutations can modify the expression of FGFR2 protein, a tyrosine-protein kinase acting as a cell-surface receptor for FGF. FGFR2 signaling induces immature cells to become bone cells during embryonic development. Accordingly, overexpression of FGFR2 can cause the bones of the skull to fuse prematurely, resulting in abnormal bone growth and facial deformities. Crouzon's syndrome may be transmitted as an autosomal dominant genetic condition or may arise consequent to a de novo mutation. Epigenetic mechanisms, such as a decrease in miR-338 with consequent increases in FGFR2 expression and osteoblast differentiation, may also underlie the development of Crouzon's syndrome.

\section{References}

1. Rutland P, Pulleyn LJ, Reardon W, Baraitser M, Hayward R, Jones B, Malcolm S, Winter RM, Oldridge M, Slaney SF, Poole MD. Identical mutations in the FGFR2 gene cause both Pfeiffer and Crouzon syndrome phenotypes. Nature genetics. 1995;9(2):173. 
2. Li ZL, Chen X, Zhuang WJ, Zhao W, Liu YN, Zhang FX, Ha RS, Wu JH, Zhao C, Sheng XL. FGFR2 mutation in a Chinese family with unusual Crouzon syndrome. International journal of ophthalmology. 2016;9(10):1403.

3. Bowling EL, Burstein FD. Crouzon syndrome. Optometry J Am Optometric Assoc. 2006;77(5):217-22.

4. Ke R, Lei J, Ge M, Cai T, Yang J, Wu Y, Mu X. Severe Meningeal Calcification in a Crouzon Patient Carrying a Mutant C342W FGFR2. J Craniofac Surg. 2015;26(2):5579.

5. Altintas AG, Aksoy FG, Altintas SC, Midillioglu IK, Duman S. Evaluation of findings in Crouzon's syndrome. Orbit. 1999;18(4):247-59.

6. Profitt WR, Fields HW, Sarver DM. Contemporary orthodontics. 5th ed. St. Louis: Mosby Elsevier; 2012.

7. Kaushik A, Bhatia H, Sharma N. Crouzon's Syndrome: A Rare Genetic Disorder. Int J Clin Ped Dent. 2016 Oct;9(4):384.

8. Rocco FD, Renier D, Marchac D, Arnaud E. Syndromic Craniosynostosis. Youmans Winn Neurolog Surg. 2017;194:1570-80.

9. Giordano BP, Tuli SS, Ryan SF, Stern M, Tuli SY. Crouzon syndrome: Visual diagnosis. Journal of Pediatric Health Care. 2016 May 1;30(3):270-3.

10. Helms JA, Schneider RA. Cranial skeletal biology. Nature. 2003;423(6937):326.

11. Hlongwa P. Early orthodontic management of Crouzon Syndrome: A case report. Journal of maxillofacial and oral surgery. 2009 Mar 1;8(1):74-6.

12. Cohen MM. Craniosynostosis and syndromes. J College Physicians Surg Pak. 2009 Jun;19(5):318-20.

13. Cha JY, Maddileti S, Mitin N, Harden TK, Der CJ. Aberrant receptor internalization and enhanced FRS2-dependent signaling contribute to the transforming activity of the fibroblast growth factor receptor 2 IIIb C3 isoform. J Bio Chem. 2009;284(10):622740.

14. Goos JA, van den Ouweland AM, Swagemakers S, Verkerk AJ, Hoogeboom AJ, van Veelen ML, Mathijssen IM, van der Spek PJ. A novel mutation in FGFR2. Am J Med Gen Part A. 2015 Jan 1;167(1):123-7.

15. Glaser RL, Jiang W, Boyadjiev SA, Tran AK, Zachary AA, Van Maldergem L, Johnson D, Walsh S, Oldridge M, Wall SA, Wilkie AO. Paternal origin of FGFR2 mutations in sporadic cases of Crouzon syndrome and Pfeiffer syndrome. Am J Hum Gen. 2000;66(3):768-77.

16. Wilkinson CC, Manchester DK, Keating RF, Ketch LL, Winston KR. Syndromic craniosynostosis, fibroblast growth factor receptor 2 (FGFR2) mutations, and sacrococcygeal eversion presenting as human tails. Child's Nervous System. 2012;28(8):1221-6.

17. Itoh N, Ornitz DM. Evolution of the Fgf and Fgfr gene families. TRENDS in Genetics. 2004 Nov 1;20(11):563-9.

18. Ornitz DM, Marie PJ. Fibroblast growth factor signaling in skeletal development and disease. Genes Develop. 2015;29(14):1463-86.

19. Sher S, Cole P, Kaufman Y, Hatef DA, Hollier L. Craniosynostotic variations in syndromic, identical twins. Annals Plastic Surg. 2008;61(3):290-3.

20. Ornitz DM, Itoh $\mathrm{N}$. The fibroblast growth factor signaling pathway. Wiley Interdisciplinary Rev: Develop Bio. 2015;4(3):215-66. 